\title{
Las prácticas en extensión universitaria para los alumnos de grado. Integración de docencia, investigación y extensión
}

\author{
The practices in university extension for the students of \\ degree. Integration of teaching, research and extension
}

\author{
Elizabeth Jorge \\ Universidad Nacional de Córdoba \\ Argentina \\ eli21jorge@gmail.com \\ Lucía Muñoz \\ Universidad Nacional de Córdoba \\ Argentina \\ luciam_08@hotmail.com \\ Andrea García \\ Universidad Nacional de Córdoba \\ Argentina \\ andrea11_g@hotmail.com \\ Macarena Guzmán \\ Universidad Nacional de Córdoba \\ Argentina \\ macarenaguzman07@gmail.com
}

Recibido: 06/05/2019 Aceptado: 10/09/2019

Resumen. Se presentan las reflexiones originadas a partir de una práctica de estudiantes de grado en un proyecto extensionista universitario, en el marco de la Cátedra de Psicología Clínica (Facultad de Psicología, Universidad Nacional de Córdoba). Teniendo en cuenta que la extensión no forma parte de la currícula de formación del alumnado, las autoras de este escrito proponen una serie de cavilaciones en torno a la articulación entre la docencia, la investigación y la extensión. 
Revista Universidad en DiÁlogo • Vol. 10, N. 1, Enero-Junio, 2020 • 11-29

ISSN 2215-2849 • EISSN: 2215-4752

URL: http://www.revistas.una.ac.cr/index.php/dialogo/index CORREO ELECTRÓNICO: universidadendialogo@una.cr

DOI: https://doi.org/10.15359/udre.10-1.1

Se describe el proyecto extensionista desarrollado hasta el momento y el diseño de la propuesta para la participación de los alumnos de la carrera de Psicología. Para el estudiantado, los procesos procuraron la articulación teórico-práctica del quehacer del psicólogo clínico en el marco de un proyecto de vinculación entre la universidad y la sociedad. De esta manera se promovió el avance en la apropiación de conceptos relativos a la propia disciplina. Además, se entrenaron en la utilización de dos herramientas fundamentales para el ejercicio del rol profesional: la observación y el registro, ambos necesarios para la reflexión posterior.

Para las docentes autoras de este escrito, se exponen las implicancias de esta propuesta de práctica en torno a dos ejes: el acuerdo sobre el tipo de formación de los estudiantes y qué objetivos se buscaba alcanzar (articulación docencia y extensión), y la evaluación del impacto de esta experiencia en el alumnado (articulación con la investigación). En el primer eje se trabajó el concepto de educación experiencial, mientras que en el segundo se abordó el tema de las competencias.

Palabras clave: prácticas, población estudiantil universitaria, docencia, extensión.

\begin{abstract}
In this paper, we present reflections from an internship of undergraduate students during a university extension project, within the framework of the Chair of Clinical Psychology (Faculty of Psychology, National University of Córdoba). Taking into account that the extension is not part of the curriculum of the students' training, the authors of this paper propose a series of thoughts about the articulation between teaching, research, and extension.

The paper describes the extension project under development, and the design of the proposal for the participation of students enrolled in the Psychology program. For the students, the processes sought the theoretical-practical articulation of the clinical psychologist work within the framework of a project linking the university with society. Thus, the appropriation of concepts related to the discipline itself was promoted. Besides, the students received training in using two fundamental tools for the exercise of the professional role: observation and registration, both necessary for further reflection.

The authors of this paper explained the implications of this internship proposal around two axes: the agreement on the type of student training and the objectives to be achieved (articulation teaching and extension), and the evaluation of the impact of this experience on students (articulation with research). The first axis focused on working on the concept of experiential education; the second one focused on addressing the competences topic.
\end{abstract}

Keywords: internships, university students, teaching, extension. 
URL: http://www.revistas.una.ac.cr/index.php/dialogo/index

CORREO ELECTRÓNICO: universidadendialogo@una.cr

DOI: https://doi.org/10.15359/udre.10-1.1

\section{La extensión en el ámbito universitario y la invitación a los alumnos de grado ${ }^{1}$}

Las actividades del docente universitario suponen una articulación entre la enseñanza, la investigación y la extensión. De esta manera se conjugan la preparación de futuros profesionales, la producción de conocimientos y la vinculación de la universidad con la comunidad donde se encuentra inserta (López, 2012).

En relación con la extensión, puede decirse que es la función menos claramente definida, más diversamente entendida y más vulnerable a los cambios de las situaciones políticas internas y externas de la universidad (Pacheco, 2012). Sin embargo, hay acuerdo en sostener el espacio de la extensión como aquel que permite el encuentro entre la universidad y la sociedad (Arocena, 2010; Corvalán de Mezzano, 2010; Pacheco, 2012; Menéndez et al., 2013).

De esta manera, se puede afirmar que:

La extensión no sólo es un proceso de formación integral a través del cual se co-resuelven problemáticas sociales definidas, con el aporte del conocimiento producido por la investigación, la reflexión y la crítica, sino que constituye un aporte fundamental a la planificación de la enseñanza formal universitaria y para la orientación, tematización de la investigación científica. Es, por lo tanto, la función que sirve de guía política a la institución y que garantiza la pertinencia social del trabajo de la universidad. (Pacheco, 2012, p. 22)

Canelones (2010) plantea que las universidades actúan como agentes sociales con el propósito de redefinir conceptos y prácticas referentes a su rol de creador de conocimientos. Estas se orientan a la transformación tanto de su propia práctica académica como de su entorno social. Para tal fin, es preciso que las diferentes entidades educativas pongan en marcha la construcción de diferentes escenarios en los que circulen las oportunidades de crear y divulgar el saber, de modo que llegue a los contextos en los cuales se interviene.

La universidad, en la implementación de la extensión universitaria, es considerada una entidad competente para "crear, adquirir y transmitir conocimiento" (Mayo y Lank, 2002, citados en Canelones, 2010, p. 56), y a su vez, es capaz de transformarse y modelarse a nuevas ideas y conceptualizaciones.

Parte de las ideas aquí expresadas fueron expuestas por las autoras en el VIII Encuentro Nacional y Latinoamericano "La Universidad como objeto de investigación. La Reforma Universitaria entre dos siglos”. Mayo del 2017. Universidad Nacional del Litoral (Argentina). 
Revista Universidad en DiÁlogo • Vol. 10, N. ${ }^{\circ}$ 1, Enero-Junio, 2020 • 11-29

ISSN 2215-2849 • EISSN: 2215-4752

URL: http://www.revistas.una.ac.cr/index.php/dialogo/index Correo ElECTRÓNICO: universidadendialogo@una.cr

DOI: https://doi.org/10.15359/udre.10-1.1

En la extensión universitaria se valora el compromiso social, ya que las universidades se acercan a las problemáticas del contexto y aportan a la creación de nuevas conceptualizaciones y reflexiones, lo cual produce un impacto en el aprendizaje.

La triada docencia, investigación y extensión habilita una configuración más integral en torno a la formación de los profesionales que transiten en la universidad. Es decir, otorga un plus a aquellas personas que indaguen, dentro de sus recorridos profesionales, la búsqueda de cambios en el contexto, según los emergentes que los convoque a realizar intervenciones.

Siguiendo al Núcleo de Directores de Cultura y Extensión, se puede decir que la

Extensión universitaria, como función básica institucional, se interrelaciona con la docencia y la investigación y con su entorno socioeconómico y cultural: ocupa un lugar preponderante en el desarrollo de las políticas universitarias al vincular de manera permanente y dinámica a la Universidad con la sociedad, y estimular en el estudiante la creatividad y la capacidad crítica, la confrontación teórico-práctica y al dar utilidad social al conocimiento que se imparte en las aulas. De este modo, la universidad se constituye en un recurso valioso de transformación y progreso social. (NDCE, 2002, p. 2)

Por lo tanto, la extensión universitaria promueve la construcción de saberes actualizados y pertinentes en pos de favorecer el crecimiento humano y profesional comprometido con la transformación del contexto social. Esta coyuntura entre el saber y el contexto concede la posibilidad del desarrollo y de la expansión de la extensión universitaria como educación continua, acción social y como divulgación y transferencia de conocimiento.

Esto último está vinculado con la responsabilidad de la extensión universitaria en la formación de profesionales comprometidos y comprometidas con su universidad y con los intereses de la comunidad. Impulsando, de esta forma, la participación conjunta, promocionando la investigación y la docencia en beneficio de la comunidad y colaborando en generar soluciones a los problemas del entorno, desde el conocimiento diverso y heterogéneo.

Por ello, la construcción del conocimiento lleva en sí misma la posibilidad de transformar críticamente esa praxis construida socialmente en conjunto con la universidad. Esto encierra el desafío de intentar comprender las complejas interacciones existentes entre la enseñanza y la transformación social. 
La extensión universitaria le ofrece al alumnado encontrarse con situaciones y experiencias que enseñan nuevas formas de construir el pensamiento, así como también poner en tensión aquellos conocimientos adquiridos. Ante textos y contextos que desarrollen y develen diferentes formas de abordar la realidad, que habiliten la creación, deconstrucción y reconstrucción de conocimientos que permitan explicitar modos creativos en el ser, saber y hacer personal y profesional. La extensión universitaria como actividad de acción social conduce a modificaciones en la manera de concebir, diseñar y gestionar el currículo, y, por ende, en el aprendizaje.

Desde el año 2009, un equipo de docentes de la Cátedra de Psicología Clínica de la Facultad de Psicología (Universidad Nacional de Córdoba) ${ }^{2}$ desarrolla un proyecto extensionista llamado Orientación y Contención a los Alumnos Tutores. Este proyecto surge a partir de la demanda realizada por dos escuelas preuniversitarias de la ciudad de Córdoba. Los alumnos tutores son estudiantes adolescentes de ambas escuelas que asisten a los últimos años (quinto, sexto) o séptimos años, y voluntariamente participan realizando apoyo escolar a niños de dos escuelas primarias públicas. En el vínculo que se establece entre los niños y los alumnos tutores prima la confianza y el lazo afectivo. A partir de ello, se suscitan ciertas emociones, malestares y sentimientos en los adolescentes que necesitan ser expresados y elaborados con ayuda de otros. Es por ello que se solicita la intervención de la Cátedra, para poder brindar un espacio donde esto pueda ser abordado.

El servicio que se ofrece, desde el equipo interviniente de la Cátedra, a los alumnos tutores consiste en a) facilitar un espacio que permita expresar las fantasías, creencias, temores, ideas y expectativas previas al desempeño del rol de alumno-tutor; b) identificar las características personales que facilitarían el desempeño del rol; c) desarrollar estrategias que permitan la construcción del vínculo con el niño; d) acompañar la elaboración de ansiedades básicas que la práctica genera; e) recoger las expresiones de los alumnos tutores en relación con lo que le significó la experiencia; y f) brindar un espacio de contención de las emociones generadas por la separación y disolución del vínculo.

La práctica de Orientación y Contención a los Alumnos Tutores se desarrolla durante un año lectivo, desde marzo hasta los primeros días de diciembre. Consiste en asistir a cada una de las escuelas preuniversitarias demandantes, en un total de cuatro reuniones que son entendidas como "unidades funcionales".

Esta materia forma parte del Plan de Estudios de la Licenciatura y el Profesorado en Psicología, y se ubica en el cuarto año de la formación. 
Revista Universidad en DiÁlogo • Vol. 10, N. ${ }^{\circ}$ 1, Enero-Junio, 2020 • 11-29

ISSN 2215-2849 • EISSN: 2215-4752

URL: http://www.revistas.una.ac.cr/index.php/dialogo/index Correo ElECTRÓNICO: universidadendialogo@una.cr

DOI: https://doi.org/10.15359/udre.10-1.1

Es decir, que cada una de ellas se desdobla en dos o más encuentros, dependiendo de las necesidades del grupo de alumnos tutores que se acompaña. Los ejes sobre los que se desarrollan las actividades pensadas para cada una de estas reuniones son cuatro, a saber: a) construcción del rol de tutor, b) expresión de fantasías, temores y creencias en torno al rol a desempeñar, c) seguimiento, y d) cierre y evaluación con los alumnos tutores.

Durante el año 2016 se decidió elaborar una propuesta de práctica para los alumnos de la Cátedra en el marco del proyecto extensionista que se venía desarrollando. Debe considerarse que la extensión no forma parte del currículo de la carrera y, por lo tanto, gran parte de los alumnos universitarios de nuestra facultad transitan una formación curricular obligatoria, y solo algunos pocos añaden un voluntariado opcional y otros desconocen las prácticas de carácter extensionista que brinda la facultad.

Se formuló, entonces, una primera experiencia que funcionaría como prueba piloto. Se convocó a un número reducido de alumnos (diez en total) para que la presencia de ellos en los espacios de encuentros con los alumnos tutores no fuera invasiva para los adolescentes.

El diseño de esta oferta de práctica llevó al equipo coordinador a reflexionar sobre las implicancias en el rol docente y en la formación de los estudiantes de grado. Se pueden señalar principalmente dos ejes que guiaron las reflexiones: por un lado, una puesta en común sobre cómo se concebía la formación de los estudiantes y qué objetivos se buscaba alcanzar. Por otro, cómo se evaluaría el impacto de esta experiencia en la formación de los alumnos. La primera posibilitó la reflexión sobre la articulación extensión-docencia, mientras que la segunda permitió la articulación con la investigación. En los apartados que siguen se debaten cada uno de estos ejes.

\section{La docencia y la extensión: una propuesta en torno a la educación experiencial}

Formarse en una carrera relacionada con la salud implica desarrollar determinados aprendizajes, habilidades y destrezas que se pondrán en juego en las intervenciones profesionales en un futuro. Es por ello que se consideran importantes las formas y características del aprendizaje de los estudiantes de grado, así como el alcance y el aprovechamiento de los recursos y características personales (Esguerra Pérez y Guerrero Ospina, 2010). En este sentido es que se consideró la concepción de educación experiencial, postulada por John 
Dewey en 1899 para definir el marco donde los estudiantes de la Cátedra de Psicología Clínica harían su participación.

La educación experiencial, según Camilloni (2013), puede ser definida como:

Una clase particular de aprendizajes, una estrategia de enseñanza con enfoque holístico que está destinada a relacionar el aprendizaje académico con la vida real. Con ese fin, propone al alumno realizar actividades en las que, a partir de esa conexión con la práctica, se le requiere que ponga a prueba en situación auténtica, es decir, de la vida real, las habilidades y los conocimientos teóricos que posee, que evalúe sus consecuencias, enriquezca esos conocimientos y habilidades, identifique nuevos problemas y fije prioridades en cuanto a las urgencias de su solución. (p. 15)

La educación experiencial ofrece estrategias educativas, efectivas, que ponen el énfasis en la educación centrada en el estudiante, de manera interactiva con el medio, a fin de construir conocimiento. Este tipo de educación se vale de múltiples elementos, entre los cuales están los estímulos físicos y sensoriales, junto a la inteligencia, con el objetivo de promover la capacidad de aprender a aprender (Department for Education and Skills, 2006 en Romero, 2010).

De la educación experiencial deviene, entonces, el aprendizaje experiencial. Este ofrece una oportunidad única para conectar la teoría y la práctica. Smith (2001 en Romero, 2010) señala que uno de los rasgos particulares del aprendizaje experiencial es que involucra al alumno en una interacción directa con aquello que se está estudiando, en lugar de una mera "contemplación" o descripción intelectual. No obstante, este autor enfatiza en que no basta la experiencia para asegurar el aprendizaje, sino que este está íntimamente ligado a un proceso de reflexión personal en el que se construye significado a partir de la experiencia vivida.

Dewey (1938, en Romero, 2010) reivindica el potencial que ofrece la experiencia para promover conocimiento, comprendiendo que los alumnos aprenden cuando encuentran significado en su interacción con el medio. Este autor distingue distintas fases, aplicables a la educación experiencial, entre ellas: experiencia concreta, reflexión, conceptualización abstracta y aplicación. Por lo tanto, de acuerdo con esa perspectiva, el aprendizaje que obtienen los educandos se inicia a partir de una experiencia concreta, la cual es interpretada a través de la reflexión y la conceptualización. 
Revista Universidad en DiÁlogo • Vol. 10, N. ${ }^{\circ}$ 1, Enero-Junio, 2020 • 11-29

ISSN 2215-2849 • EISSN: 2215-4752

URL: http://www.revistas.una.ac.cr/index.php/dialogo/index Correo ElECTRÓNICO: universidadendialogo@una.cr

DOI: https://doi.org/10.15359/udre.10-1.1

Durante el proceso de educación experiencial se manifiesta un entramado de interrelaciones multidireccionales, las cuales versan sobre el entorno, el docente y el alumno. Es clave el rol que desarrolla el docente, en tanto que delimita un contexto que aporte una experiencia rica en estímulos de aprendizaje y ha de incentivar la curiosidad y el interés del alumno, favoreciendo su capacidad de reflexión, de conceptualización y de aplicación de conocimiento. En este proceso, el estudiante es animado a experimentar y a aplicar su creatividad e ingenio para explorar el contexto, desarrollando conocimiento.

En el proceso enseñanza-aprendizaje, el apuntalamiento de fomentar una práctica de educación experiencial contribuye a la adecuación de ciertos contenidos específicos, los cuales se ofrecen de modo reflexivo a fin de establecer relaciones entre el conocimiento y el contexto. Estos se ajustan a las necesidades comunitarias específicas, en las que los adolescentes realizan sus actividades de tutor.

Sigmon (1999, citado en Figueroa, 2008) delimita que existe una correspondencia entre la metodología de enseñanza y el aprendizaje, a través de la cual los adolescentes desarrollan y ejercitan habilidades por medio del servicio a sus comunidades y escuelas. Esta enseñanza y aprendizaje experiencial les permite a los jóvenes comprometerse en tareas importantes y asumir roles significativos y desafiantes en la diversidad de lugares por donde circula esta dualidad. El fin al cual se aproxima este tipo de educación es incorporar ciertos dinamismos que implican historicidad y vivencias tanto individuales como grupales.

Para ello se precisa un vínculo estrecho entre los objetivos pedagógicoacadémicos y las particularidades sociocomunitarias donde se realiza la tutoría. Esto se debe a que al ser una propuesta innovadora permite mejorar la calidad de los estudios, así como recibir una respuesta participativa a una necesidad social que aporta a la "transformación" de las personas y de la sociedad. Para Benner (s/a pp 64, citado en Figueroa, 2008), "la experiencia es un requisito para ser experto", la cual es coherente en apoyar y entregar valor a las experiencias de los jóvenes, lo cual promueve y estimula el avance del conocimiento.Romero (2010) describe que la capacidad de aprender a aprender, en el marco del aprendizaje experiencial, demanda la habilidad para aprovechar todos los sentidos, tales como el oído, el olfato, la vista, el tacto y el gusto, los cuales están en interacción con el medio para construir conocimiento; esta habilidad se incrementa si además se utilizan los estímulos físicos que se reciben para aprender. 
Desde esa perspectiva, se defiende el valor del aprendizaje experiencial para promover la capacidad de aprender a aprender (Department for Education and Skills, 2006, citado en Romero, 2010). Es decir, el aprendizaje experiencial ofrece la posibilidad concreta de conectar teoría con práctica. Esto se refleja cuando el educando se encuentra con el desafío de responder a una amplia variedad de situaciones reales, y es en estas donde se consolida en él un conocimiento significativo, contextualizado, transferible y funcional, produciendo un incremento en su capacidad de aplicar lo aprendido.

Retomando una expresión de Smith (2001, citado en Romero, 2010), uno de los rasgos característicos del aprendizaje experiencial es que involucra, compromete e implica al educando tanto en la interacción con el contenido intelectual como con el proceso de reflexión personal. Es a partir de este proceso que se construye el significado de la experiencia, como consecuencia del contacto con el contexto, y es por medio de este mismo proceso que los jóvenes logran adquirir la capacidad de transferir el nuevo conocimiento a otras situaciones.

En relación con la práctica ofrecida a los alumnos, y en función de las fases de la educación experiencial, se diseñó una propuesta que incluyó, en un primer momento, una instancia previa de capacitación donde se trabajaron ejes tales como: qué es la extensión universitaria, la presentación del proyecto, las técnicas a utilizarse en la práctica (observación, registro, reflexión, sistematización), la mirada clínica en el espacio educativo, entre otros. Luego de estos encuentros, los alumnos efectuaron la experiencia de ingresar en el campo, acompañando a las docentes en la implementación del proyecto extensionista. Es decir, ejercitaron el rol de observadores no participantes en los encuentros con los alumnos tutores. Para ello, se los presentó al grupo de adolescentes, y se aclaró que tomarían notas de lo que aconteciera en esa reunión.

En un segundo momento, en el ámbito universitario, se llevó a cabo una supervisión de los estudiantes de la cátedra. En este espacio se pudieron trabajar las diferencias entre sus ideas previas con aquello vivenciado. Pudieron reflexionar sobre a) su rol de observador no participante, b) las posibilidades reales de registrar todo lo que acontece en un grupo y su dinámica, c) el registro contratransferencial, que se suscita inevitablemente en el encuentro/ escucha con otros, d) sus habilidades y limitaciones para el trabajo en un futuro con adolescentes, y e) las necesidades de seguir formándose y entrenarse en determinadas herramientas. 
En un tercer momento, se les pidió un trabajo de reflexión y articulación teórico-práctica. Los estudiantes pudieron preguntarse sobre el marco teórico de referencia, no al modo de "encajar teoría y práctica", sino empezando a contemplar qué de la teoría responde a lo observado. También se abrieron camino en la búsqueda de conceptualizaciones que les permitieran comprender lo experimentado y acercar unas posibles respuestas a sus preguntas. Asimismo, pudieron comprender de manera más global la implicancia del rol profesional en un proyecto extensionista, donde cada una de las intervenciones que se hacen son para favorecer el funcionamiento de una propuesta que nos conecta con las necesidades de un grupo de la sociedad.

En este sentido, se plantea la posibilidad de un entrecruzamiento entre la teoría y la práctica, así como también la puesta en práctica de los conocimientos, las habilidades y las capacidades del alumno en una situación concreta de aprendizaje. Esto puede ejemplificarse con algunos comentarios de los alumnos que participaron de la práctica:

Podemos mencionar la posibilidad de experimentar el rol de observador no participante. Este rol implicó mucho esfuerzo para anotar todo aquello que escuchábamos, observábamos y sentíamos (alumna 1).

La posibilidad de entrelazar todo aquello de lo que habíamos sido partícipes al tomar notas y los aportes teóricos que creíamos que nos ayudaban a explicarlo. De esta manera se pudo dar cuenta de que la teoría refleja la práctica, pero no en completud, porque siempre va a haber una parte de la misma que, sin experiencia previa, es imposible conocer (alumna 2).

Como se puede apreciar en los dichos de estos estudiantes, las reflexiones que realizan de su práctica se hace teniendo en cuenta tanto la experiencia previa como aquella que está constituyéndose a partir de su participación. Los ejemplos citados en los párrafos anteriores buscan mostrar el modo de aprendizaje mediante la educación experiencial. Considerando que esta es una experiencia subjetiva, que permite la permanente interacción entre la acción, el pensamiento y la reflexión.

Siguiendoa Camilloni(2013), la educación experiencialadmite un procesoreflexivo que no es sin la participación del propio sujeto que construye conocimiento: "Es un proceso por el cual una vivencia o un conjunto de vivencias se convierte en "experiencia" y ésta en un aprendizaje reconocido como tal" (p. 15). 
Se podría pensar entonces este espacio de práctica para los alumnos de la Cátedra como un lugar donde puedan aplicar las habilidades adquiridas a lo largo de todo el proceso de enseñanza-aprendizaje de un modo reflexivo. Además, se habilita este espacio como transformador, ya que la intervención del alumno, en su conocimiento activo, no es sin consecuencias en su subjetividad y, además, en el entorno social que habita.

En esta permanente estimulación de la puesta en práctica de su capacidad de observación y registro, se permite que el alumno despliegue su creatividad, de manera que le posibilite insertarse en otros ámbitos, de los cuales quizás nunca haya formado parte. En relación con lo anterior, y siguiendo a Tommasino (2008), se puede aludir a los procesos de enseñanza y aprendizaje que se dan fuera del aula, tal como esta práctica:

Los procesos de enseñanza y aprendizaje se generan y operan en terreno, junto a la gente, partiendo de los problemas que la gente tiene, intentando junto con ella encontrar alternativas, esta situación reconfigura el acto educativo y fundamentalmente redimensiona el poder que circula en los diferentes actores del proceso. Aquí operan varios sub-procesos que desestructuran el acto educativo tal cual lo conocemos intra-muros o en el aula. (p. 6)

Estos procesos de enseñanza y aprendizaje extramuros posibilitan diversas reconfiguraciones tanto en el docente como en los estudiantes. En cuanto al rol docente, este se transforma desde el mismo momento en que se cambia el lugar-aula por la realidad misma. Es desde esta realidad donde se generan preguntas en boca de gente que no será evaluada y que oficia como un evaluador de lo que el docente sabe, ya que demanda soluciones y explicaciones que no están contextualizadas o regladas, que no están encuadradas por el aula (Tommasino, 2008).

Por otra parte, en cuanto a la posición de los alumnos, también se da una reconfiguración, dado que se posiciona también como un actor social y como un futuro profesional. Es preciso, entonces, desde el rol docente, valorar la experiencia y las competencias de quien aprende, y, además, percibir el potencial de productividad que proporcionan los aprendizajes experienciales en los alumnos. El siguiente apartado trata sobre las competencias adquiridas en el marco de la práctica realizada por los estudiantes de la Cátedra. 
Revista Universidad en DiÁlogo • Vol. 10, N. ${ }^{\circ}$ 1, Enero-Junio, 2020 • 11-29

ISSN 2215-2849 • EISSN: 2215-4752

URL: http://www.revistas.una.ac.cr/index.php/dialogo/index CORREO ELECTRÓNICO: universidadendialogo@una.cr

DOI: https://doi.org/10.15359/udre.10-1.1

\section{La investigación y la extensión: ¿Qué competencias adquirieron los alumnos con su participación?}

Uno de los aspectos que interesaba investigar con los estudiantes era el impacto de esta práctica en su formación profesional. Para ello se eligió indagar sobre las competencias que habían podido poner en práctica en las diferentes actividades implicadas. Siguiendo a González, Vilche y Knopoff (2013), se pueden definir las competencias asociándolas con la aptitud, la idoneidad, la destreza y el dominio sobre alguna situación en particular.

Para Díaz Barriga (2003, en González et al., 2013), el concepto de competencias se relaciona con los términos aptitudes y habilidades. El primero de ellos da cuenta de diversas disposiciones de cada individuo; mientras que el segundo remite a la pericia que se ha desarrollado a partir de tales disposiciones. Es un concepto que debe considerarse desde una visión integral, abarcando las capacidades del sujeto que actúa en un contexto social (González et al., 2013). Bolívar (2005) expresa que el término competencia está alcanzando una gran profusión en la última década, tanto en los textos profesionales como en cuanto a las competencias de aprendizaje a promover en los alumnos mediante la enseñanza. En general, "competencia profesional es la pericia, aptitud o idoneidad" (pp. 6-7).

Parafraseando a Álvarez Pérez (2012), se puede afirmar: no es suficiente que los estudiantes aprendan muchos conocimientos y conceptos. Sino que se hace necesario también que dominen habilidades de vida y competencias generales aplicables a diversidad de situaciones y contextos en los que una persona se desenvuelve. Y esto solo se logra a través de una orientación continua y organizada, de modo que se potencie tanto la dimensión académica como la personal, social y profesional.

Los elementos esenciales de las competencias se pueden enumerar como: (1) son características o atributos personales: conocimientos, habilidades, aptitudes, rasgos de carácter, conceptos de uno mismo; (2) están causalmente relacionados con ejecuciones que producen resultados exitosos, se manifiestan en la acción; (3) son características subyacentes a la persona que funcionan como un sistema interactivo y globalizador, como un todo inseparable que es superior y diferente a la suma de atributos individuales; (4) logran resultados en diferentes contextos. Estos cuatro elementos son esenciales para el concepto de competencia y no puede darse la misma si falta uno de estos (Fernández, 2005). Funcionaría como un sistema de engranaje donde las partes permanecen interrelacionadas y el cambio en una de ellas impactaría inevitablemente en el resto. 
Tal como se mencionó anteriormente, en el marco de la propuesta a los alumnos, se buscó indagar sobre las competencias que habían ejercitado. Para ello, al finalizar la práctica, se les pidió que completaran el instrumento "Autoevaluación de competencias en el ejercicio del rol". Se trabajó individualmente con el "Cuestionario de autoevaluación de competencias profesionales en la tutoría" (Cocolotl, 2014; Flores y Cocolotl, 2015) que fue adaptado especialmente para esta propuesta (Jorge y Guzmán, 2016).

El instrumento original estaba dirigido a tutores para su automonitoreo. Consistía en 110 ítems para valorar el nivel de dominio, producto de la autoevaluación. Está organizado en cuatro dimensiones: (1) trabajo con los alumnos, (2) trabajo con familia, (3) vinculación con la escuela, y (4) recursos profesionales individuales. Cada reactivo tiene cuatro opciones de respuesta: (1) no lo poseo y necesito ayuda, (2) no lo domino y busco recursos, (3) lo domino, y (4) lo domino y puedo enseñarlo. Quien completa el cuestionario debe elegir la opción que más se acerque a su nivel de desempeño logrado hasta el momento. Se sugiere, asimismo, que quien lo responda lo haga de la manera más objetiva y honesta posible, dado que los resultados son beneficiosos principalmente para sí mismo.

Para el trabajo que se presenta en este artículo, se adaptó el cuestionario, ya que se consideró que es un instrumento creado especialmente para la observación de los tutores (población destinataria del proyecto extensionista que da marco a la práctica de los alumnos de grado). Por otra parte, en relación con los estudiantes de grado incluidos, era importante que pudieran reflexionar sobre las habilidades puestas en juego durante la experiencia. Es por ello que solo se utilizó la cuarta dimensión del cuestionario original: "Recursos profesionales individuales", con un total de diecisiete ítems. El cuestionario de autoevaluación es entendido como una valoración reflexiva del tutor en términos del nivel de dominio en el trabajo realizado por el alumno sobre los recursos profesionales con los que cuenta para automonitorearse en la tarea que realiza (Cocolotl, 2014).

El nivel de dominio de las competencias autoevaluadas osciló entre "lo domino" y "lo domino y puedo enseñarlo". Esto significa que los estudiantes pudieron retomar, identificar e intervenir en la práctica de acuerdo con los requerimientos establecidos por el equipo docente. Planearon actividades con los conocimientos revisados en la capacitación previa a la práctica. Diseñaron su articulación teórico-práctica final con un buen desempeño. Mostraron actitudes respetuosas, participativas y comprometidas con la experiencia de la práctica, en sus distintas instancias (capacitación, asistencia a las instituciones, 
Revista Universidad en DiÁlogo • Vol. 10, N. ${ }^{\circ}$ 1, Enero-Junio, 2020 • 11-29

ISSN 2215-2849 • EISSN: 2215-4752

URL: http://www.revistas.una.ac.cr/index.php/dialogo/index CORREO ELECTRÓNICO: universidadendialogo@una.cr

DOI: https://doi.org/10.15359/udre.10-1.1

supervisiones). En este sentido, se puede decir que la experiencia ofrecida por el equipo promovió la reflexión del alumno, el desarrollo de competencias y una formación integral desde tres vertientes de desarrollo: académica, personal y profesional. Todo esto supone para el estudiante un papel más activo en su propio proceso de aprendizaje.

Describiendo con mayor detalle, se pudieron detectar que aquellas competencias donde se perciben con fortalezas son "analizar diferentes perspectivas conceptuales sobre problemáticas con los adolescentes del Proyecto Extensionista", "respeto los puntos de vista que no coinciden con mi opinión", "muestro interés y gusto por trabajar con adolescentes", "muestro paciencia ante situaciones inesperadas o no contempladas con los adolescentes del Proyecto Extensionista", "muestro flexibilidad para adaptarme a situaciones que estén fuera de lo común en el Proyecto Extensionista", "mantengo la confidencialidad de lo observado en la práctica y la supervisión", "poseo una actitud crítica y constructiva hacia las diferentes propuestas que se realizan en el Proyecto Extensionista", "poseo interés por la búsqueda de respuestas que satisfagan las problemáticas presentadas en el Proyecto Extensionista", "muestro interés por continuar de manera autónoma el desarrollo de mis competencias profesionales", "muestro preocupación por superar mis carencias en cuanto a conocimientos y habilidades".

Considerando estas competencias autopercibidas por los mismos estudiantes, se podría afirmar que la propuesta del equipo se acerca a una formación sobre los conocimientos científicos y técnicos, con capacidad de aplicarlos en diversos contextos, integrándolos con sus propias actitudes y valores en un modo propio de actuar, tanto personal como profesionalmente (Villa Sánchez y Villa Leicea, 2007).

Por otra parte, aquella competencia en la que percibieron que deben seguir trabajando para mejorarla o adquirirla fue "analizo mi sentir y actuar respecto a los entrevistados". A partir de estos resultados, el equipo docente interviniente tiene el desafío de generar, en las próximas propuestas de prácticas, las condiciones necesarias que permitan fortalecer y ejercitar aquellas competencias que se "consideran aprehendidas", a la vez que posibilitar el desarrollo de otras con las que todavía no cuentan o lo hacen de manera muy incipiente.

Asimismo, si se tiene en cuenta el concepto de competencia profesional, es decir, "la capacidad productiva de un individuo que se define y mide en términos del desempeño de una profesión y refleja los conocimientos, habilidades, destrezas y actitudes necesarias para realizar las tareas propias de su profesión con eficiencia y calidad" (Rodríguez, Rojas y Vera, s. f., p. 
2), puede decirse que los estudiantes pudieron poner en juego los recursos intelectuales, los conocimientos disponibles, realizando un ejercicio de sus competencias de modo más flexible y profundo (Contreras, 1999, citado en Barrón, 2009).

Todo esto se corresponde con aquello que se sostiene en cuanto a la educación universitaria: se dice que tiene, entre sus objetivos fundamentales, formar profesionales competentes al servicio de la ciudadanía. Bolívar (2005) expresa que "las instituciones de educación superior deben contribuir a que los futuros profesionales desarrollen una visión y sentido ético, que pueda guiar su práctica y refleje en sus acciones un conjunto de valores (responsabilidad, solidaridad, sentido de la justicia, servicio a otros)" (p. 6).

Como señala Hortal (2002, citado en Bolívar, 2005), para que un profesional se legitime como experto la competencia profesional no basta. Para lograrlo, el profesional necesita asumir los compromisos que comparte con sus colegas de profesión. Es decir, apoderarse de la responsabilidad de tratar de proporcionar competentemente las prestaciones y los servicios específicos según los estándares de excelencia que en cada contexto se espera de cada tipo de servicio profesional.

Por otra parte, se hace necesario en este punto hacer algunas puntualizaciones sobre el proceso de reflexión personal que se habilitó hacia el final de la práctica, mediante el cuestionario implementado.

No debe olvidarse que la reflexión personal contribuye a la autoevaluación, la cual exige planificar, percibir, recordar, pensar, analizar, sintetizar, inferir, valorar y tomar decisiones, entre otros procesos. Es necesaria para valorar las fortalezas y áreas de oportunidad en el proceso de aprendizaje durante la práctica (Cocolotl, 2014). En el caso de los alumnos incorporados a la práctica de grado, la autoevaluación se llevó a cabo al finalizar la experiencia. Siguiendo a Rueda (2010), se puede decir que la autoevaluación se constituye en una herramienta que permite un conocimiento sistemático de la situación analizada, de la orientación para las acciones futuras y la construcción de sentido en la toma de decisiones.

Tal como sostienen Flores, Otero y Lavalleé (2010), las personas que se autoevalúan asumen un compromiso con su aprendizaje y reflexionan sobre las implicaciones futuras de la formación profesional. La autoevaluación permite tomar conciencia sobre las propias limitaciones, con lo cual se activan mecanismos para superarlas y proponer acciones innovadoras para mejorar la práctica (Cocolotl, 2014). 
Revista Universidad en DiÁlogo • Vol. 10, N. ${ }^{\circ}$ 1, Enero-Junio, 2020 • 11-29

ISSN 2215-2849 • EISSN: 2215-4752

URL: http://www.revistas.una.ac.cr/index.php/dialogo/index Correo ElECTRÓNICO: universidadendialogo@una.cr DOI: https://doi.org/10.15359/udre.10-1.1

Es así que analizar los procesos y hacer una evaluación general de las fortalezas y los límites que encuentran a la hora de articular la teoría con la práctica son claves en el proceso de aprendizaje. La valoración es un elemento que permite el desarrollo profesional y potencia la calidad formativa de los estudiantes de grado. Se puede pensar, entonces, que el aprendizaje comienza a la hora de la primera propuesta y el compromiso de llevar a cabo la tarea. Es un acercamiento que los ayuda a pensarse en terreno, a ir construyendo una identidad profesional, a equivocarse y a asumir responsabilidades.

\section{Consideraciones finales}

Tal como sostienen Villa Sánchez y Villa Leicea (2007), las universidades siempre han tenido en su misión el desarrollo de una formación integral de sus estudiantes. En este artículo se buscó reflexionar sobre la incorporación de los estudiantes de grado para llevar a cabo prácticas en el marco de un proyecto extensionista.

Si se piensa en la palabra extensión, se puede atribuir que hay algo que se extiende, que va más allá de la universidad, pero que a la vez comparte con ella. La extensión no como algo que está "fuera de", sino como práctica que puede funcionar articulándose con la docencia y la investigación.

La posibilidad de incorporar a estudiantes avanzados de una carrera de grado a un proyecto extensionista permite, primero, que conozcan y puedan interesarse por las prácticas extensionistas que proporcionan un encuentro entre la universidad y la comunidad. Segundo, insertarse en otro ámbito de aprendizaje que permita la adquisición de herramientas para construir su identidad profesional. Esta identidad no está solamente determinada por la inserción de alumnos a diferentes prácticas, sino que también se apoyará en conocimientos previos, en la educación experiencial y en procesos de enseñanza-aprendizaje, entre otros. De esta manera se logra una integración de los conocimientos y las experiencias en las trayectorias de cada alumno.

Por otra parte, se procuró que los estudiantes logren una articulación teórico-práctica del quehacer del psicólogo clínico en el marco del proyecto extensionista. A través de las distintas actividades propuestas, se procuró la apropiación y revisión de los conceptos propios de la disciplina. Además, se entrenaron en la utilización de herramientas fundamentales para el ejercicio profesional: la observación y el registro, los cuales facilitaron la reflexión posterior. Es decir, se buscó integrar compromiso, conocimiento y puesta en práctica, y además se promovió la reflexión ética en el ejercicio de la profesión. 
Por último, indagar sobre el impacto de la práctica en los estudiantes, mediante la evaluación de las competencias en las diferentes actividades en las que participaron, implicó un posicionamiento del equipo docente interviniente. En este sentido, se pensó a la práctica ofrecida como un espacio de formación de conocimientos científicos y técnicos, que pueden aplicarse en contextos diversos, y que promueven una integración entre los saberes, los valores y las actitudes (personales y profesionales), promoviendo el desarrollo de la identidad profesional (Barba y Alcántara, 2003). Con base en la reflexión personal que llevaron a cabo los alumnos sobre la experiencia realizada se puede inferir que, mediante esta práctica, se ha contribuido significativamente en la formación profesional.

Para finalizar, se puede decir que el equipo docente se encuentra en los albores de un camino, largo e interesante, que permitirá el diseño y desarrollo de nuevas estrategias en el trabajo con los alumnos de grado dentro de la extensión universitaria. Porque, tal como lo plantean Villa Sánchez y Villa Leicea (2007):

No se trata sólo de mejorar la preparación profesional de los estudiantes para ocupar un puesto laboral determinado, sino, principalmente, ofrecer una formación más sólida, más firme, más acorde con el enfoque de lo que debe ser una buena formación universitaria, que ayude a los estudiantes a saber, saber hacer, a convivir y a ser. (p. 17)

\section{Referencias bibliográficas}

Álvarez, P. R. (2012). Los planes institucionales de tutoría y el desarrollo de competencias en el marco del Espacio Europeo de Educación Superior: un estudio desde la perspectiva del alumnado. Perfiles educativos, 34(137), 28-45.

Arocena, R. (2010). Curricularización de la extensión: ¿porqué, cuál, cómo? Cuadernos de Extensión $N^{\circ} 1$. Universidad de la República, Uruguay.

Barba, L. y Alcántara, A. (2003, diciembre). Los valores y la formación universitaria. Reencuentro, 38, 16-23.

Barrón, M. (2009). Docencia universitaria y competencias didácticas. Perfiles Educativos. 1-13.

Bolívar, A. (2005). El lugar de la ética profesional en la formación universitaria. Revista Mexicana de Investigación Educativa.1-31. 
Camilloni, A. (2013). La inclusión de la educación experiencial en el currículo universitario. En Menéndez, G. (Comp.), Integración, docencia y extensión. Otra forma de enseñar y aprender.(1-98) Universidad Nacional del Litoral, Santa Fe.

Canelones, M. (2010). Extensión universitaria como acción social. Impacto innovador a partir de su inserción en el currículo. Revista Informe de Investigaciones Educativas, 24.

Cocolotl, D. (2014). La autoevaluación en los tutores del PAES: la construcción de un cuestionario (Tesis de Maestría). Facultad de Psicología, Universidad Autónoma de México.

Corvalán de Mezzano, A. (2010). Psicólogos institucionales trabajando. La psicología institucional en docencia, investigación y extensión universitaria (2. ${ }^{\circ}$ ed.). Buenos Aires: Eudeba Editorial.

Esguerra, G. y Guerrero, P. (2010). Estilos de aprendizaje y rendimiento académico en estudiantes de Psicología. Revista Diversitas-Perspectivas en Psicología, 6(1), 97-109.

Fernández, J. M. (2005). Matriz de competencias del docente de educación básica. Revista Iberoamericana de Educación. (ISSN: 1681-5653)

Figueroa, A. A. (2008). Ética, solidaridad y "aprendizaje servicio" en la educación superior. Acta Bioethica, 14(1), 61-67

Flores, R. y Cocolotl, D. (2015). Cuestionario de autoevaluación de las competencias profesionales en las tutorías [Inédito].

Flores, R. C.; Otero, A. y Lavalleé, M. (2010). La evolución de la perspectiva epistemológica en estudiantes de postgrado. El caso de los psicólogos escolares. Revista Perfiles Educativos, 32(130), 8-24.

González, M.; Vilche, E. y Knopoff, P. (2013). Aprendizaje fuera del aula. Aportaciones de la extensión universitaria. Segundas Jornadas de Investigación y Transferencia. Facultad de Ingeniería, Universidad Nacional La Plata.

Jorge, E. y Guzmán, M. (2016). Adaptación del Cuestionario de autoevaluación de las competencias profesionales en las tutorias, para alumnos tutores, coordinadores y estudiantes observadores [Inédito]. 
López, M. L. (2012). Extensión universitaria: situación actual y aportes metodológicos. Universidad Nacional de Jujuy, San Salvador de Jujuy.

Mayo, A. y Lank, E. (2002). Las organizaciones que aprenden. Barcelona: Gestión 2000.

Menéndez, G. (Comp.). (2013). Integración, docencia y extensión. Otra forma de enseñar y aprender. Universidad Nacional del Litoral, Santa Fe.

Pacheco, M. (2012). Reflexiones en torno a la construcción del espacio de la extensión universitaria hoy. Cuadernos de Educación, 21-30.

Rodríguez, M. A., Rojas, A. y Vera, F. (s. f.). Acción tutorial basada en competencias. Centro Universitario de Ciencias Exactas e Ingenierías, Universidad de Guadalajara.

Romero, M. (2010). El aprendizaje experiencial y las nuevas demandas formativas. Revista de Antropología Experimental, 10(especial educación 8), 89-102. Recuperado de https://revistaselectronicas.ujaen. es/index.php/rae/issue/view/184

Rueda, M. (2010). Autoevaluación y autonomía. Revista Perfiles Educativos, 32(130), 3-6.

Tommasino, H. (2008). Generalización de las prácticas integrales. Los aportes de la extensión para su implementación. Recuperado de psico.edu.uy/ sites/default/files/tommasino_practicas_integrales.pdf

Villa Sánchez, A. y Villa Leicea, O. (2007). El aprendizaje basado en competencias y el desarrollo de la dimensión social en las universidades. Educar, 40, 15-48. 